\title{
Ankle Arthrodesis Nail Combined with Locking Compression Plate to Stabilize Two-Level Pathologic Tibial Fractures
}

\author{
G. Ulrich Exner ${ }^{1 *}\left(\mathbb{0}\right.$, Gerardo Juan Maquieira $^{2}$, Natasha Forster ${ }^{3}$, Pascal A. Schai ${ }^{4}$ \\ ${ }^{1}$ Orthopaedie Zentrum Zuerich, Zuerich, Switzerland \\ ${ }^{2}$ Fuss Zentrum Hirslanden Zuerich, Klinik Hirslanden, Zuerich, Switzerland \\ ${ }^{3}$ Swiss Parc, Plastic Surgery, Zuerich, Switzerland \\ ${ }^{4}$ Luzerner Kantonspital Wolhusen, Wolhusen, Switzerland \\ Email: ^guexner@gmail.com, gerardo.maquieira@hirslanden.ch, natasha.forster@swissparc.ch, pascal.schai@luks.ch
}

How to cite this paper: Exner, G.U., Maquieira, G.J., Forster, N. and Schai, P.A. (2021) Ankle Arthrodesis Nail Combined with Locking Compression Plate to Stabilize Two-Level Pathologic Tibial Fractures. Open Journal of Orthopedics, 11, 301-307.

https://doi.org/10.4236/ojo.2021.1110028

Received: September 3, 2021

Accepted: October 17, 2021

Published: October 20, 2021

Copyright ( 2021 by author(s) and Scientific Research Publishing Inc. This work is licensed under the Creative Commons Attribution International License (CC BY 4.0).

http://creativecommons.org/licenses/by/4.0/

\begin{abstract}
Treatment of fragility fractures of the distal tibia in the elderly is demanding because of osteopenic bone quality, the delicate soft tissue cuff and frequent critical circulation. We report the case of two-level tibial pseudarthroses in an 83year-old woman caused by multiple myeloma successfully stabilized by a long ankle arthrodesis nail combined with locking compression plate osteosynthesis. This case is unique, as to our best knowledge, herein diaphyseal fractures were treated for the first time using a technique reported hitherto primarily for fragility ankle and pilon fractures.
\end{abstract}

\section{Keywords}

Pathologic Fractures of the Tibia, Plasma cell myeloma, Ankle Arthrodesis Nail, Periprosthetic Tibia Fracture, Intramedullary Nail

\section{Introduction}

Treatment of fragility fractures of the distal tibia in the elderly is demanding because of osteopenic bone quality, the delicate soft tissue cuff and frequent critical circulation. Among other indications to minimize soft tissue damage retrograde nailing has been reported as salvage for failed surgical management of ankle fractures [1]. Retrograde nailing has been extended to special indications as ankle fragility/osteoporotic, open distal tibia and talus, complex tibial pilon fractures, and aseptic non-unions [2]-[8].

We report on a case of pathologic two-level fractures of the tibia due to exten- 
sive bone destruction by a plasma cell myeloma treated using a retrograde ankle arthrodesis nail after failed plate osteosynthesis with infected soft tissue necrosis.

\section{Case Presentation}

The now 83 year old lady was diagnosed at age 77 years to have a multiple myeloma IgG kappa diagnosed on the occasion of a pathologic fracture L3 treated by corporektomy and spondylodesis. The situation was well controlled clinically under oncologic treatment for the following 5 years until she suffered a pathologic fracture of the ulna, which was treated by plate osteosynthesis and irradiation. Shortly thereafter a proximal meta-diaphysial tibia fracture distal to a total knee replacement implanted 10 years ago occurred (Figure 1).

The fracture was categorized as "periprosthetic pathologic" with stable endoprosthetic components. Computed Tomography (CT) performed for tumor staging revealed the involvement of the entire tibia with a large distal meta-diaphysial osteolysis. Stabilizing the proximal fracture zone and prophylactically the distal osteolytic zone was planned using a locking nail fixation preserving the well fixed tibial component.

After reaming forwarding the nail over the tibial isthmus was imposed by the curvature of the nail and limitations of the entry point caused by the endoprosthesis.

It was then decided to stabilize the proximal fracture only with a short medial locking compression plate LCP DePuy Synthes ${ }^{\circledast}$ (Figure 2) avoiding the risk of distal soft tissue failure.

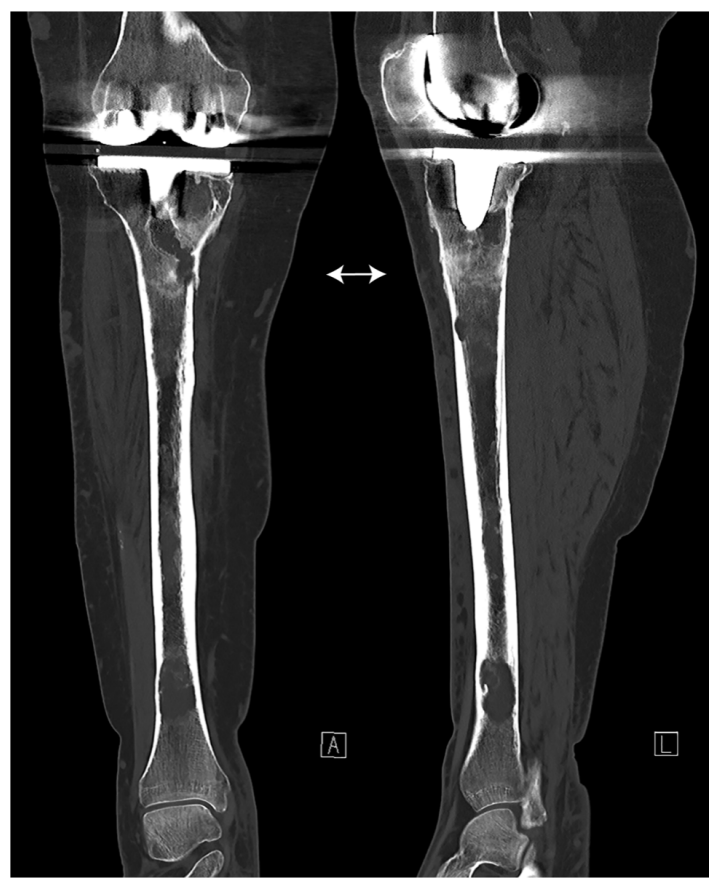

Figure 1. Coronal CT-reconstructions ap and lateral: Extensive osteolysis from the plasma cell myeloma. Fracture (arrows) through the sclerotic changes distal to the knee replacement (implanted 10 years earlier). 


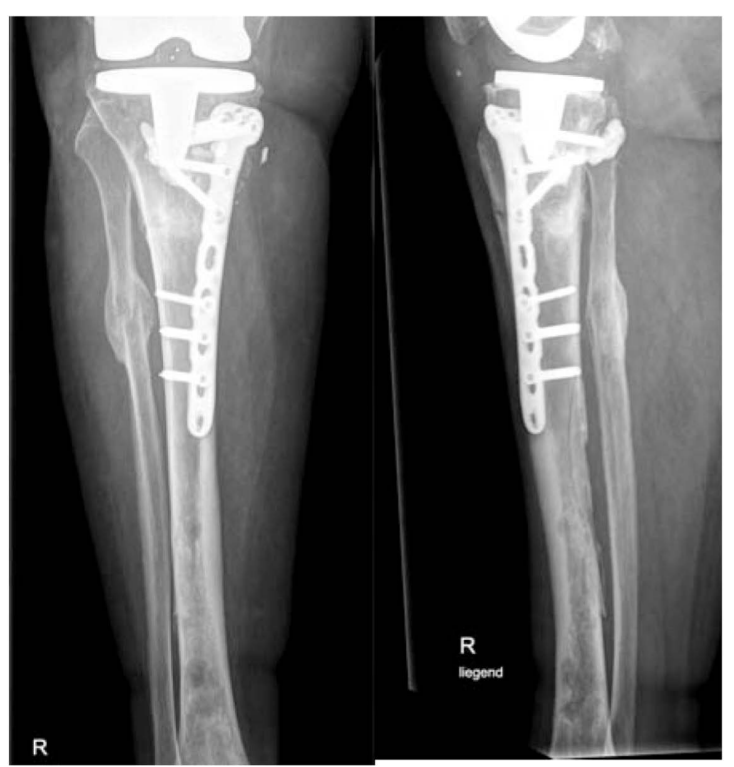

Figure 2. X-ray 4 months after plate osteosynthesis of the proximal tibia fracture with cement augmentation around the proximal screws. The former fibula fracture is unrelated to the present problem.

Postoperatively the tibia was irradiated with 30 Gy over 2 weeks. The patient could walk pain free for 4 months.

She then developed incapacitating pain caused by the fragile distal osteolysis with impending fracture. To stabilize the entire tibia the short plate was exchanged to a contoured left proximal lateral LCP DePuy-Synthes ${ }^{\circledast}$ tibia plate (Figure 3).

Whereas the proximal wound healed well skin necrosis developed distally exposing the tibial tendon with contamination of Pseudomonas aeruginosa (Figure 4). Angiography revealed vascular conditions not allowing for a free microvascular muscle transfer. Angioplasty of the Aa. poplitea, tibialis anterior and posterior, resection of the exposed tibialis anterior tendon, repeated débridements, split skin grafting, VAC-treatment, and parenteral antibiotic (Zavicefta ${ }^{\circ}$ \{ Avibactam + Ceftazidim\}) over a period of 3 months led to secondary wound healing and normalization of infective serum parameters.

The plate was never exposed and was removed after complete healing of the soft tissue cuff.

Ongoing pain at ambulation distally even following normalization of infective parameters suggested insufficiency of the fixation. At this time it was considered to change stabilization of the distal insufficiency fracture by an ankle arthrodesis nail and the proximal pseudarthrosis again by a plate. Contamination of the implants with Pseudomonas aeruginosa could not be excluded; it was therefore decided for a staged procedure. At removal of the plate screw fracture and pathologic mobility proved that insufficiency through the large osteolysis had developed (Figure 5). Culture of the sonicated plate proved continued growth of Pseudomonas aeruginosa. 

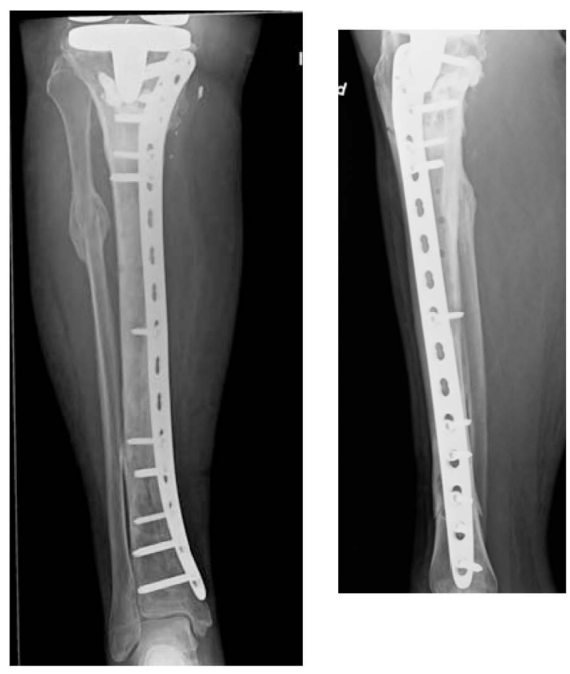

Figure 3. X-ray 2.5 months after conversion to a long plate to stabilize the distal osteolysis that has meanwhile developed.

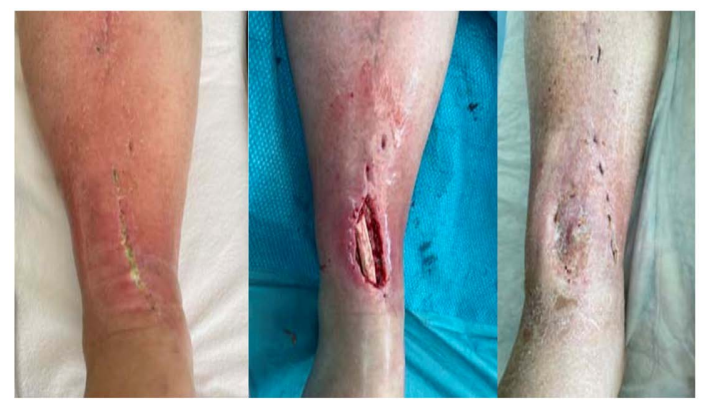

Figure 4. Wound dehiscence following insertion of the long plate (a) with exposure of the tibialis anterior tendon (b), healed after resection of the tendon, repeated debridement, VAC, split skin and antibiotics (c).

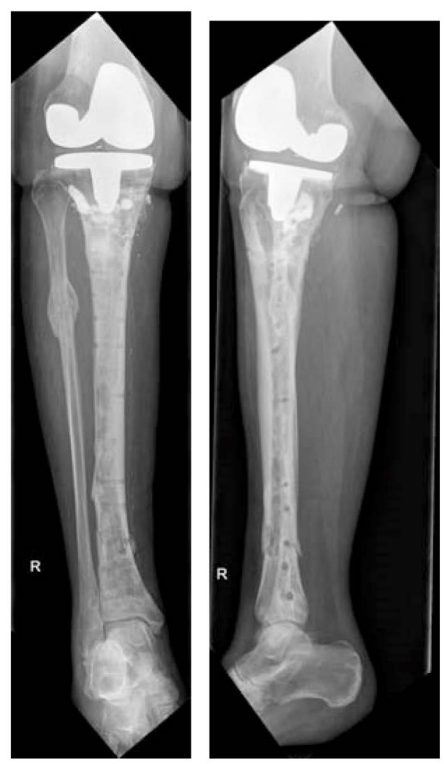

Figure 5. Standard X-ray after removal of the long plate and screws document the persistent proximal and distal tibia fracture (arrows). 
After extensive discussion with the patient including the option of amputation 3 weeks later, complete wound healing and normalization of infection parameters operative stabilization deemed feasible. Because of the two-level fracture-instability with persistent periprosthetic pseudarthrosis at the proximal meta-diaphysial zone and the pathologic distal zone, weight bearing tolerable stabilization of the entire tibial bone was mandatory.

The distal pseudarthrosis was stabilized with the $30 \mathrm{~cm}$ long T2 ankle arthrodesis nail Stryker ${ }^{\circledR}$ and the proximal persistent fracture by an overlapping DePuy Synthes ${ }^{\circledR}$ LCP tibia plate (Figure 6). The ankle arthrodesis nail was implanted according to standard operative technique. Tibio-talo-calcaneal arthrodesis was omitted to reduce additional soft tissue compromise.

No further bacterial growth from the tibia bone marrow aspirate, nor vital myeloma cells were found at pathologic examination.

Immediately postoperative the patient was comfortable at rest and having only minimal pain around the hind foot with protected weight bearing using canes. Wounds healed primarily. Healing of the fractures cannot be expected around the plasma cell myeloma following irradiation.

Since 2 months postoperative the patient walks without using canes inside her condominium, outdoors she uses canes or a rollator.

\section{Discussion}

Open Reduction and Internal Fixation (ORIF) of distal tibia fractures inherently carries the general risk of wound healing and even more in the elderly due to vascular compromise as experienced in the presented patient.

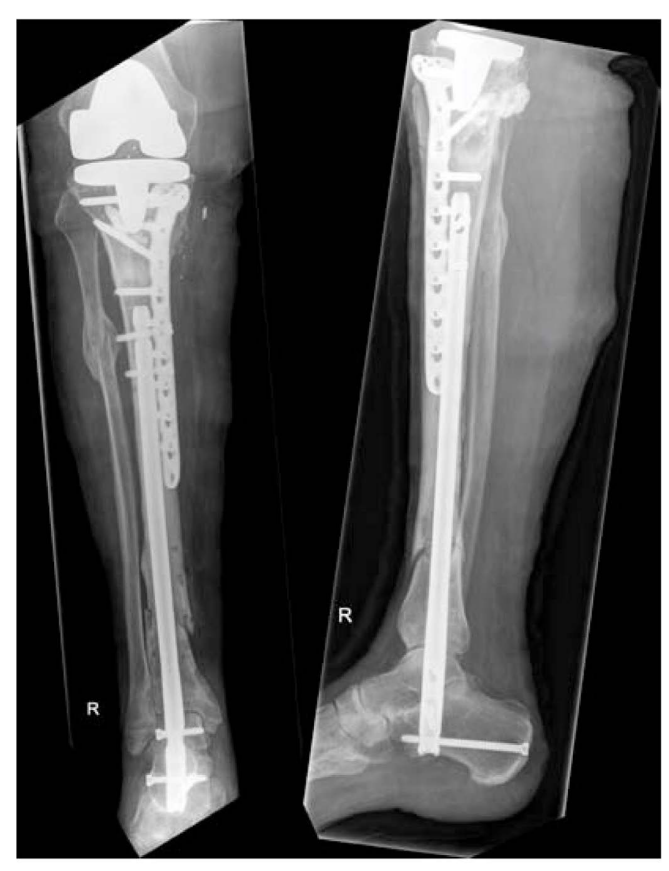

Figure 6. Postoperative X-ray documentation of the T2 ankle arthrodesis nail to stabilize the distal fracture and overlapping plate for the proximal periprosthetic fracture. 
Including the talo-calcaneal block for stabilization of ankle fractures was first reported by Duke [9] using a Steinmann pin. The first report using an intramedullary nail including the hindfoot was reported by Lemon et al. [2]. Al-Nammari et al. [6] used a retrograde femoral nail as the then available ankle arthrodesis nails were too short to cross the isthmus of the tibia. A short Stryker T2 arthrodesis nail gave a satisfactory result in the case of Hsu and Szatkowski [7]. Stryker now provides the $\mathrm{T}^{\otimes}{ }^{\otimes}$ Ankle Arthrodesis Nail with lengths up to $30 \mathrm{~cm}$ that appeared appropriate for the needs in our case allowing for anatomic position of the hind foot. Neither Al-Nammari et al. [6], Lemon et al. [2] nor Hsu et al. [7] performed tibio-talo-calcaneal arthrodesis. Lemon's case [2] continued to have excellent function after removal of the nail.

Instead of replating with the longer plate risking wound healing problems known when exposing the distal tibia we should have already considered retrograde nailing. This may have spared the patient prolonged morbidity and repeated surgeries.

\section{Conclusions}

In this patient, retrograde nailing proved to be very satisfactory. Tumor cases often present the "non-standard problems" and therefore frequently ask for "nonstandard" solutions.

Concomitant problems regarding the unique fracture patterns, neoplastic bone involvement and irradiation, and degenerative vascular changes needed multidisciplinary collaboration developing innovative ideas possibly deviating from standard concepts.

Solutions may sometimes consist in "off-label use" of "off-the-shelf" materials available for standard indications. Having used this less invasive method initially could have spared additional morbidity in the presented patient.

\section{Conflicts of Interest}

The authors declare that there is no conflict of interest regarding the publication of this article.

The patient agreed to publish her case in the presented form.

\section{References}

[1] Houshian, S., Bajaj, S.K. and Mohammed, A.M. (2006) Salvage of Osteoporotic Ankle Fractures after Failed Primary Fixation with an Ankle Arthrodesis Nail: A Report on Four Cases. Injury, 37, 791-794. https://doi.org/10.1016/j.injury.2005.08.011

[2] Lemon, M., Somayaji, H.S., Khaleel, A. and Elliott, D.S. (2005) Fragility Fractures of the Ankle. Stabilsation with an Expandible Calcanotalotibial Nail. The Journal of Bone and Joint Surgery, 87-B, 809-813. https://doi.org/10.1302/0301-620X.87B6.16146

[3] Amirfeyz, R., Bacon, A., Ling, J., Blom, A., Hepple, S., Winson, I. and Harries, W. (2008) Fixation of Ankle Fragility Fractures by Tibiotalocalcaneal Nail. Archives of Orthopaedic and Trauma Surgery, 128, 423-428. 
https://doi.org/10.1007/s00402-008-0584-Z

[4] Ochmann, S., Evers, J., Raschke, M.J. and Vordemvenne, T. (2012) Retrograde Nail for Tibiotalocalcaneal Arthrodesis as a Lim Salvage Procedure for Open Distal Tibia and Talus Fractures with Severe Bone Loss. The Journal of Foot \& Ankle Surgery, 51, 675-679. https://doi.org/10.1053/j.jfas.2012.04.015

[5] Jonas, S.C., Young, A.F., Curwen, C.H. and McCann, P.A. (2013) Functional Outcome following Tibio-Talar-Calcaneal Nailing for Unstable Osteoporotic Ankle Fracture. Injury, 44, 994-997. https://doi.org/10.1016/j.injury.2012.11.008

[6] Al-Nammari, S.S., Dawson-Bowling, S., Amin, A. and Nielsen, D. (2014) Fragility Fractures of the Ankle in the Frail Elderly Patient. Treatment with a Long Calcaneotalotibial Nail. The Bone and Joint Journal, 96-B, 817-822. https://doi.org/10.1302/0301-620X.96B6.32721

[7] Hsu, A.R. and Szatkowski, J.P. (2014) Early Tibiocalcaneal Arthrodesis Intramedullary Nail for Treatment of Complex Tibial Pilon Fracture (AO/OTA 43-C). Foot \& Ankle Specialist, 8, 220-225. https://doi.org/10.1177/1938640014548322

[8] Mosca, M., Caravelli, S., Fuiano, M., Massimi, S., Censoni, D., Grassi, A., Vocale, E., Ceccarelli, F. and Zaffagnini, S. (2020) Tibiotalocalcaneal Arthrodesis through Retrograde Nailing for the Treatment of Juxtaarticular Distal Tibia Aseptic Non-Unions: A Retrospective Study at a Minimum Follow-Up of 4 Years. Injury, 51, 1377-1381. https://doi.org/10.1016/j.injury.2020.03.039

[9] Duke, R.F.N. and Oxon, B.M. (1963) Severe Fracture Dislocation of Ankle Treated by Transarticular Steinmann Pin. The Lancet, 282, 1251-1253.

https://doi.org/10.1016/S0140-6736(63)90897-3 